\section{Questión}

Periodismo / Comunicación ISSN 1669.6581

Cambiemos y la Modernización del Estado Argentino ¿Realidad o Ficción?

María Laura Farías

Question/Cuestión, Nro.70, Vol.3, diciembre 2021

ISSN: 1669-6581

URL de la Revista: https://perio.unlp.edu.ar/ojs/index.php/question/

IICom -FPyCS -UNLP

DOI: https//doi.org/10.24215/16696581e637

\title{
Cambiemos y la Modernización del Estado Argentino
}

\section{¿Realidad o Ficción?}

\section{Cambiemos and Argentine State Modernization}

\section{Reality or Fiction?}

María Laura Farías

Instituto de Investigaciones Gino Germani, Facultad de Ciencias Sociales, Universidad

Nacional de Buenos Aires

Argentina

lic.mlfarias@gmail.com https://orcid.org/0000-0001-6701-4253

\section{Resumen}

Producto de su reciente surgimiento en Argentina y en el marco de un proceso de investigación doctoral, el artículo analiza las diferentes estrategias y dispositivos implementados por una serie de Altos Funcionarios que, habiendo sido directivos de grandes empresas, fueron designados como los principales articuladores del Plan de Modernización del Estado durante el período de gobierno de la coalición Cambiemos (2015-2019). Se buscará, 
por lo tanto, dar a conocer las características del proyecto de modernización, los principios y valores que rigieron para los empleados públicos y los dispositivos implementados que buscaron influir en la subjetividad estatal.

Palabras Clave: Argentina; management; Estado; modernización; Alta Dirección Pública

\section{Abstract}

As a result of its recent emergence in Argentina and within the framework of a doctoral research process, the article analyzes the different strategies and devices implemented by Senior Public Managers who, having been directors of large companies, were designated as the main articulators of The State Modernization Plan during the period of government of the Cambiemos coalition (2015-2019). Therefore, it will seek to publicize the characteristics of the modernization project, the principles and values that governed public employees and the implemented devices that sought to influence state subjectivity.

Key words: Argentina; management; State; modernization; Senior Public Management

\section{Introducción}

La modernización del Estado es un tema del que ningún espacio social se priva debatir. Ahora bien, modernizar el Estado puede tener diferentes significaciones dependiendo de la cosmovisión que atraviese cada una de esas esferas (académicas, políticas, entre otras). En Argentina, y enfocándonos en la esfera política, la coalición Cambiemos -que gobernó el Estado Nacional de 2015 a 2019- no fue la excepción, con una única característica ciertamente novedosa: por primera vez en la historia política del país, un gobierno liderado por un empresario (Mauricio Macri) definía como parte de su gabinete a personas que respondían a lógicas diferentes a las de antaño.

Para un mayor entendimiento, es importante traer a colación algunas cuestiones previas. A fines del siglo XX surgió, en Latinoamérica, una nueva forma de gestión de lo estatal: la doctrina de la Nueva Gestión Pública. En ella se establecieron diferentes principios rectores basados en flexibilidad de la organización, la orientación a resultados y la satisfacción 
del cliente, estructuras más horizontales que estimularan la creatividad en sus empleados, entre otras, para poder administrar y gestionar al Estado y poder convertir a esa organización en un ente más productivo y eficaz (en otros términos, modernizándose). En el caso de Argentina, si bien la modernización del Estado puede rastrearse desde épocas anteriores (Farías, 2021:50), los años '90 tuvieron un marcado protagonismo en la vinculación con los dispositivos de esa doctrina, pero influida por el neoliberalismo, lo cual implicó una noción de gobierno centrada en la lógica empresarial (Reynares, 2017).

En ese período en particular, las empresas transnacionales comenzaron a radicarse en el país, y producto de las decisiones tomadas por el Poder Ejecutivo de esa época, se llevó a cabo un proceso de privatización de diversas empresas públicas y de modificaciones sustanciales dentro del aparato estatal. Para lograr implementar esas acciones modernizadoras, el management fue de gran utilidad para los objetivos del gobierno de turno: se contrataron una serie de expertos provenientes de grandes empresas privadas para que, a modo de consultores, acompañaran el proceso de adquisición de una empresa pública o la modificación de ciertos procedimientos internos de las reparticiones estatales. Un ejemplo de este fenómeno fue el de la empresa pública ENTel (1): la multinacional France Telecom implementó diferentes estrategias al tomar las operaciones en la zona sur del país, entre las cuales surgieron tres mutaciones epistemológicas sobre los trabajadores estatales: pasar de una noción de «compañero de trabajo» a «cliente interno», de «trabajador» a «colaborador» y de «pleno empleo» a «empleabilidad» (Pierbattisti, 2013:70).

Habiendo transitado el país por etapas previas tendientes a buscar la renovación del Estado, durante el período 2015 a 2019 ocurrió algo ciertamente excepcional: el management se adentró por completo en un nuevo espacio social(1), el Estado. En el escenario político que atravesó el país en esos años, se implementaron diferentes estrategias de gestión donde se intentó transformar el Estado Nacional en aparente vinculación a los postulados de la cosmovisión managerial y los lineamientos de la Nueva Gestión Pública. La diferencia radicaba en una característica novedosa: parte de las acciones de este plan estratégico implicaron la ubicación de actores (con determinadas características) en puestos políticos dentro de la estructura de cada una de las instituciones estatales. Estos actores provenían del mundo privado, mayormente, como representantes de los ideales manageriales (2) y como tales, su 
objetivo consistió en brindar «nuevos aires», trayendo «renovados» conocimientos y una filosofía del mundo de los negocios que permitiría adaptar el Estado a esta perspectiva.

Para estos individuos (Vommaro, 2017), el formar parte de la estructura estatal significó un desafío personal que les permitió dejar una huella en la historia de la nación, siendo reconocidos como individuos que actuaban para construir un país mejor. Esta suerte de rentabilidad moral compensó la escasa retribución económica que los managers percibieron por ejercer una función jerárquica en el Estado. Utilizando el fantasma de la chavización como pánico moral (3), estos managers se concibieron como emprendedores morales que buscaron brindar un servicio público como la forma más alta de caridad.

A través de la designación de estos directivos en cargos de Alta Dirección Pública (puestos con función jerárquica que van desde Director Nacional hasta Ministro inclusive), se buscó reformar la capacidad de gestión del Estado, pero a nivel institucional. Desde la manera que tenía de vincularse con los ciudadanos, se intentó redefinir la cultura del servicio público buscando consolidar nuevos hábitos y comportamientos a lo largo y a lo ancho de su estructura, afines a las prácticas de empresas transnacionales.

Por lo tanto, y considerando esta novedosa injerencia directa del management en un espacio social completamente antagónico, se indagarán aquí cuáles han sido las estrategias implementadas en el marco del plan modernizador del período. Se prestará especial atención a los dispositivos implementados por los managers que ocuparon cargos jerárquicos en el Estado, para así conocer su instrumentación y de la forma en que buscaron influir en la subjetividad estatal.

\section{El mundo del "management"}

Una de las principales preguntas que surgen es la siguiente: ¿Qué es el management? Acorde a lo desarrollado por Luci (2012a), puede ser considerado como una profesión, una forma de gestión institucional, el objeto de una disciplina de estudio o la conducción de grandes corporaciones. En referencia al primer tipo, se lo puede vincular con el rol que ocupan quienes dirigen una empresa (managers), o bien cómo se manejan las organizaciones tanto públicas 
como privadas, o como parte de las ciencias en gestión de las empresas o al cuerpo directivo de las grandes empresas. No obstante, como bien menciona la autora, el management sería mucho más que eso: sería una ideología dominante en esta era, como un modelo de éxito social. A través de sus prácticas discursivas, el management sería la forma que toma el espíritu del capitalismo para ser internalizado y transmitido (Boltansky y Chiapello, 2002), posibilitando lo que algunos autores denominan como el pasaje hacia una sociedad managerial (Aubert y de Gaulejac, 1993).

Por lo anteriormente dicho, se entiende que el management involucra una serie de acciones, pensamientos, categorías y conductas que expresan su manera de entender y concebir al mundo. Y esto incluye también cómo concebimos a las personas. En relación con la cotidaneidad organizacional, las organizaciones necesariamente, para sobrevivir, tuvieron que modificar sus estrategias de manejo de los recursos humanos para adaptarse a las nuevas corrientes imperantes. Esto implicó reemplazar las antiguas metodologías por aquellas donde predominara el otorgamiento de responsabilidad y reconocimiento, promoviéndose un sentimiento de compromiso e involucramiento para con ella (Boltansky y Chiapello, 2002). El aporte de los trabajadores al sistema productivo ya no podía pensarse bajo los términos tradicionales de eficiencia, por lo tanto, las organizaciones debieron pasar de un sistema de calificaciones tradicional a un sistema de gestión por competencias donde se establecieran los parámetros, características y conductas esperables para el mundo managerial. Estas guías establecidas, que buscan orientar, conducir y normativizar las relaciones humanas en pos del adoctrinamiento y el crecimiento hacia posiciones jerárquicas, han sido la forma que tomó el managment como gramática (Luci, 2012a).

Los sujetos, por lo tanto, se convertirían en un producto de esta gramática, que propone una cierta economía moral que interviene en la constitución de categorías y principios justificativos (Luci, 2012a). Pero al mismo tiempo que producto, lo más interesante es que se convierten simultáneamente en productores (Aubert y de Gaulejac, 1993) dado que reproducen esas mismas concepciones, categorías e idearios manageriales.

\section{Crear un "manager"}


Partiendo de la noción de habitus (D’hers, 2013), entendemos que tanto las prácticas manageriales como la percepción de los managers son una expresión de la posición social que ocupan dentro de la estructura organizacional. La sociedad managerial, entonces, configura y determina los modos de percibir y las emociones de los sujetos que la integran.

¿Cómo lograr que las personas se involucren de manera tal que al mismo tiempo se conviertan en promotoras de aquello que las condiciona?

Las organizaciones, para darle forma a sus managers, deben implementar estrategias para poder penetrar en la psiquis y que lograr que representen, en cuerpo y alma, el espíritu del management. Estas estrategias tomarán la forma de dispositivos (4) para poder regular las sensaciones y consisten en procesos de selección, clasificación y elaboración de las percepciones socialmente determinadas y distribuidas (Scribano, 2012).

A sabiendas que el motor del funcionamiento psíquico de un directivo es la pasión (Aubert y de Gaulejac, 1993), las organizaciones manageriales implementan una serie de dispositivos para potenciar y controlar esa sensación, que si bien al sujeto se le presenta como una paradoja (5), a estas organizaciones les es sumamente beneficiosa. Estos dispositivos los autores los identifican según sea explícitos o implícitos.

Como parte del primer tipo, aparecen:

- $\quad$ Estrategias de formación y capacitación;

- $\quad$ Sistemas de evaluación y dirección por objetivos;

- Los estímulos positivos de naturaleza tanto financiera como no financiera.

En cuanto al segundo tipo, encontramos los siguientes:

- $\quad$ Cada día un poco más: los individuos deben superarse de manera constante, siendo cada vez más efectivos y ágiles en la gestión cotidiana;

- $\quad$ Gestión basada en lo implícito: se le pide al sujeto que realice más acciones sin que esas disposiciones se exterioricen en algún espacio; 
- $\quad$ Dirección por la sublimación: realizar alabanzas o resaltar virtudes, al mismo tiempo que se le brinda una tarea u objetivo exigente;

- $\quad$ Competencia constante: los sujetos nunca tienen estabilidad en sus puestos y los demás funcionan como amenaza, promoviéndose la constante comparación entre ellos.

Por lo tanto, estando expuesto a la acción cotidiana de estos dispositivos, el hombre managerial es moldeado y finalmente encasillado. Esos cuerpos son adaptados para sentir acorde a las diferentes condiciones de existencia del capital corporal (D'hers, 2013). Por lo tanto, bajo el principio de la excelencia y la meritocracia, el management se presenta, entonces, como un dispositivo disciplinario tanto de poder como de articulación de prácticas de subjetivación, dado que implica la implementación de prácticas donde los sujetos operan por sobre otros y además sobre sus propios modos de sentir (Zangaro, 2011).

\section{El management: impulsor de una subjetividad moderna}

Considerando las nuevas tecnologías implementadas en el Estado Nacional en ese período (6), se pone en evidencia que se buscó generar un entorno que promoviera los valores de agilidad, eficiencia y eficacia de sus procesos, pero en el marco de un paradigma que puso en el centro a los ciudadanos y no a los procesos en sí. Cada uno de los cambios introducidos implicó, por lo tanto, el reemplazo de tareas repetitivas y rutinarias por sistemas inteligentes, originalmente ejecutadas por empleados públicos. Estas modificaciones redefinieron los parámetros de competencias (en términos de habilidades, actitudes y conocimientos) que los trabajadores debían poseer para poder continuar sirviendo al Estado Nacional. Como bien mencionó el director del INAP en una entrevista, la función del empleado público ya no estaría vinculada al mero cumplimiento de una función mecánica o burocrática, sino que por su rol en ese nuevo período debía "dar respuesta al derecho de un ciudadano», con lo cual se necesitaría poder «explorar respuestas para problemas nuevos o viejos, a buscar ayuda externa (...), y a salir del espacio de confort» (Bellomo, 2017:4). Esta forma de concebir las competencias del trabajador estatal, por lo tanto, distarían de las características valoradas en épocas anteriores (Farías, 2021), siendo entonces necesario que la identidad del ser estatal 
también evolucionara hacia comportamientos que se vinculen con la innovación, la agilidad y la apertura necesarias para adaptarse al nuevo paradigma.

Para ilustrar la criticidad que tenía el poder influir en la subjetividad estatal en este período, resulta relevante traer a colación las palabras del entonces Ministro de Modernización Ibarra. En diversos espacios, su discurso hace referencia al Estado como una institución al servicio del ciudadano y la relevancia de formar al personal estatal en la modernización de los procesos, ya que:

Son las personas, aprovechando las posibilidades que ofrecen las Nuevas Tecnologías, el verdadero motor de esta transformación que está poniendo el Estado, de una vez por todas al servicio de la gente. (Ibarra, 2017:11).

Por lo tanto, considerando la relevancia que poseía la transformación del recurso humano en la transformación hacia un Estado moderno, se indagarán cuáles han sido las estrategias implementadas en el marco del plan modernizador del período. Se prestará especial atención a las acciones de implementadas por los managers que ocuparon cargos jerárquicos, cómo se instrumentaron y de qué manera se ha buscado influir en la configuración de la subjetividad estatal.

\section{Metodología}

En relación con los propósitos que buscaron abordarse en este trabajo, se llevó a cabo un estudio de carácter cualitativo, exploratorio y no experimental a través de la aplicación de entrevistas a un total de ocho trabajadores de la "Alta Dirección Pública" dentro de los Ministerios Nacionales de Producción y Hacienda.

Considerando la estructura configurada durante el período, el cual fue analizado minuciosamente por Castellani (2017), y que durante el año 2018 se realizó una reestructuración de las reparticiones estatales nacionales (7), se optó por las dos reparticiones estatales mencionadas anteriormente debido a la concentración de mayor cantidad de perfiles 
técnicos y jerárquicos con experiencia laboral previa en el ámbito privado al momento de la realización de las entrevistas, considerando las fusiones en los distintos Ministerios:

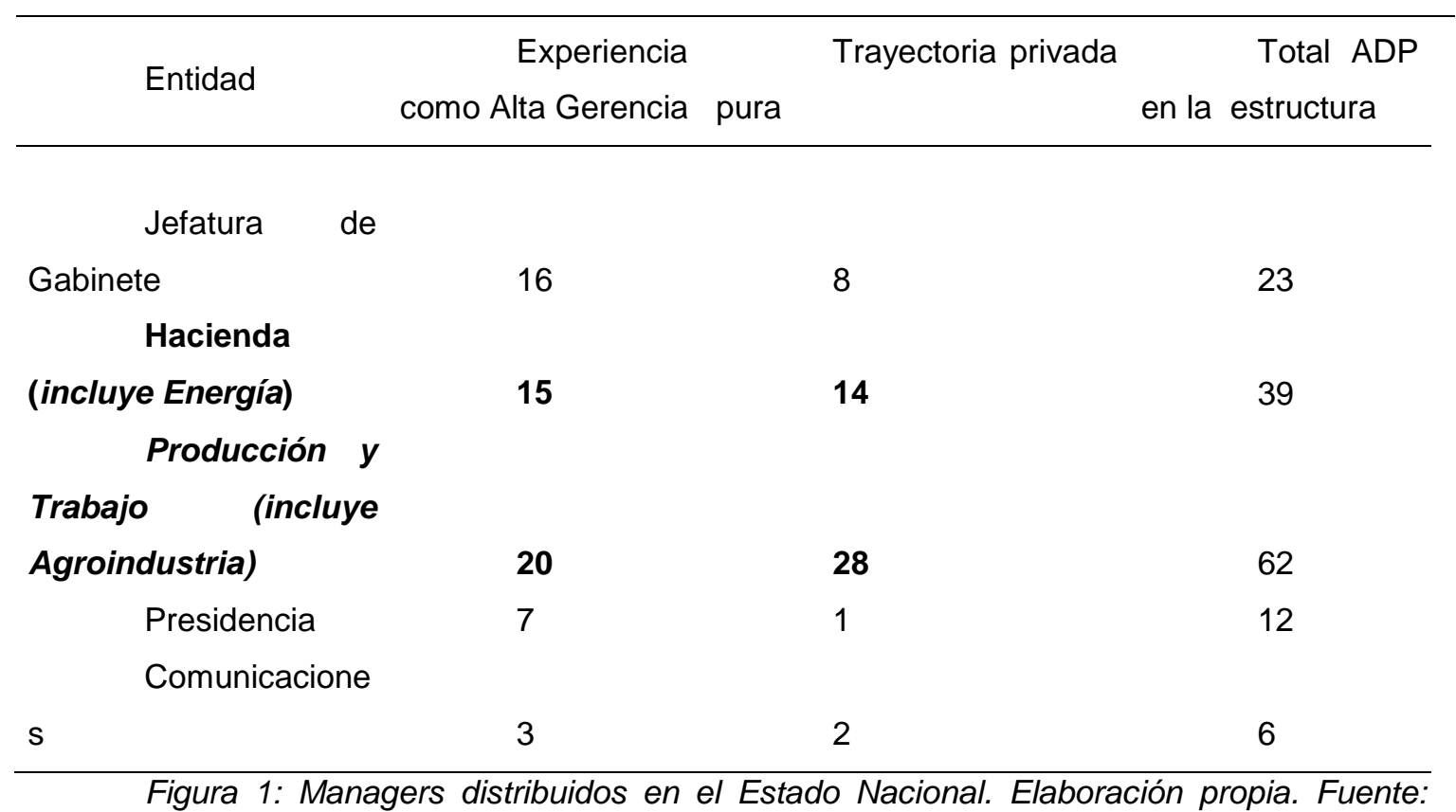

Castellani (2017)

Las condiciones que los entrevistados debían cumplir en la muestra consistían en que ocuparan un cargo jerárquico dentro de esas estructuras ministeriales (Director Nacional, Subsecretario, Secretario, Jefe de Gabinete y/o Ministro), que hubieran ingresado en el período de gobierno de Cambiemos y que hubieran ocupado previamente cargos jerárquicos en organizaciones privadas. Los encuentros se llevaron a cabo en las oficinas de los ministerios entre mayo y octubre 2019 y para los mismos se trabajó con un conjunto de guías de pautas semi-estructuradas (Vasilachis de Gialdino, 2007:71). Considerando la representatividad del cargo ejercido, su vinculación con el Poder Ejecutivo y el cumplimiento estricto de las condiciones de la muestra, se utilizó un muestreo de casos tipo (Hernández Sampieri et al., 2006). 
La muestra obtenida posee una edad desde 32 hasta 60 años, con un promedio de 46 años. Con marcado predominio del sexo masculino, se abarcó la totalidad de los puestos especificados: Ministro (13\%), Jefe de Gabinete (13\%), Secretario (13\%), Subsecretario (38\%) y Director Nacional (25\%).

\begin{tabular}{lc}
\hline Femenino & $12 \%$ \\
Masculino & $88 \%$ \\
\hline
\end{tabular}

Figura 2: Distribución por sexo.

Si bien todos cumplieron con la característica de ser graduados universitarios en ciencias tradicionales del país (Derecho, Ciencias Económicas e Ingeniería), tres de ellos obtuvieron su título de grado en una institución pública. En cuanto a estudios de posgrado, seis lo obtuvieron en escuelas de negocios de instituciones educativas privadas (8).

\begin{tabular}{lc}
\hline Grado & $25 \%$ \\
Posgrado & $75 \%$
\end{tabular}

Figura 3: Estudios universitarios máximos alcanzados.

\begin{tabular}{ll}
\hline Pública & $38 \%$ \\
Privada & $63 \%$
\end{tabular}

Figura 4: Institución universitaria del título de grado. 
Respecto de la experiencia laboral dentro del ámbito privado, poseen un promedio de 14 años de experiencia, ejerciendo cargos jerárquicos en diferentes momentos del recorrido laboral.

\begin{tabular}{lc}
\hline $0-5$ & $12,5 \%$ \\
$6-10$ & $25 \%$ \\
$11-20$ & $50 \%$ \\
& $12,5 \%$ \\
\hline Más de 20 & Figura 5: Experiencia laboral en ámbito privado (en años).
\end{tabular}

Adicionalmente, los cargos que ocupaban en la estructura estatal abarcaron la totalidad de las condiciones requeridas.

Asimismo, este tipo de técnica se complementó con entrevistas informales con informantes clave (trabajadores sin cargo jerárquico, en ambos Ministerios) que permitieron obtener más información respecto de las características de la población que representó la muestra.

Finalmente se implementó, como estrategia cualitativa complementaria, análisis de archivo (basado en la revisión de documentos oficiales emitidos a nivel gobierno Resoluciones, Decretos y Decisiones Administrativas- relacionados con la modernización del Estado), análisis bibliográfico y análisis a nivel hemerográfico, utilizando periódicos impresos y digitales.

\section{Del trabajador estatal al servidor público}


En relación con los diferentes propósitos que el gobierno de Cambiemos expresó respecto de la concepción y manejo de los recursos humanos del Estado (Farías, 2020), y también considerando los objetivos de la investigación, el material recolectado en las entrevistas y el marco teórico adoptado, el estudio llevado a cabo permitió concebir la emergencia de una serie de ejes de análisis. En primer lugar, surgió un eje relacionado a los distintos dispositivos implementados para entrenar y desarrollar a los trabajadores estatales, de manera tal de lograr vehiculizar sus esfuerzos en pos del principio de eficiencia; en segundo lugar, dispositivos relacionados con las estrategias de compensación (tanto monetarias como no monetarias) para encausar los comportamientos de los trabajadores en relación a los principios establecidos; por último, un tercer eje que enfatiza la relevancia del ambiente físico como potenciador de la motivación laboral.

En apartado en particular se expondrán los resultados obtenidos, considerando las diversas estrategias implementadas en las dos reparticiones relevadas. Adicionalmente, para mayor claridad y sustentación de las ideas principales obtenidas, se expondrán extractos de entrevistas para ilustrar el desarrollo.

\section{Eje 1: capacitación y formación}

Parte del principal accionar del plan modernizador implicó la renovación del Instituto Nacional de Administración Pública. Esta institución posee como objetivo el «formar funcionarios públicos, perfeccionando y actualizando sus conocimientos, de acuerdo a las necesidades de capacitación del Sector Público y a las exigencias de las carreras establecidas en los Estatutos y escalafones respectivo" (art. 3, Ley 20.173), por lo que en relación a este primer eje, las acciones vinculadas con la capacitación y la formación de los trabajadores públicos se manifestaron como las únicas posibles de ser instrumentadas puertas adentro a modo de premio:

Yo creo que la capacitación es el único premio que vos podes dar o la única herramienta que vos tenés, porque tenés una inflexibilidad salarial enorme, casi todo el salario es fijo depende lo que hagas, depende de tu antigüedad más que nada (Funcionario de Hacienda) 
En cuanto a los contenidos, desde la perspectiva de los Altos Funcionarios, las actividades formativas fueron consideradas como útiles en cuanto a la facilitación del conocimiento o gestión de las operaciones o de los sistemas que operaban. No obstante, el entrenamiento en habilidades que hicieran énfasis en un aspecto más actitudinal que operacional no fueron mencionados en la gran mayoría de las entrevistas. Un único caso mencionó que estos aspectos de la personalidad se desarrollaban de manera interna y no centralizada.

La percepción que existió por parte de estos funcionarios es que esas estrategias formativas fueron insuficientes para lograr un cambio en la actitud en los trabajadores estatales. Esta percepción de insuficiencia de las estrategias de este dispositivo será una constante en los relatos de los entrevistados

Eje 2: recategorizaciones, promociones y premiaciones.

La concepción inicial sobre el trabajador público previo a ocupar un cargo como Alto Funcionario, (al igual que la concepción previa sobre el Estado), no estaba exenta de negatividad: se lo vinculaba con características como defectuoso y con muchas limitaciones, distinguiéndose un prototipo de empleado público igual al personaje interpretado por Antonio Gasalla (9) (Farías, 2021). En algunos casos muy limitados existieron como pre-concepto percepciones más positivas del rol. Con el paso del tiempo en el ejercicio del rol, permitió que sugiera la posibilidad de una reconceptualización, lo cual les brindó la posibilidad de sentir que «no todo estaba tan perdido»:

Después tenés un montón de gente ávida por adoptar estas nuevas prácticas porque lo viven como un enriquecimiento personal. (Funcionario de Hacienda)

Habiendo diferenciado a los trabajadores que continuaban sus funciones bajo el paradigma anterior contra aquellos dispuestos a poder alinearse a las nuevas competencias requeridas en el proceso de modernización, el principal desafío que se les planteó a los 
entrevistados fue cómo poder generar, a nivel de la gestión cotidiana de las personas, un entorno promotor de la meritocracia.

En ambas reparticiones, aparecieron dos tipos de estrategias utilizadas: las unidades retributivas y los reconocimientos no monetarios, los cuales podrían ser pensados como estímulos positivos tanto financieros como no financieros dentro de los dispositivos explícitos que se utilizan en las organizaciones manageriales.

\section{Las Unidades Retributivas}

Las UR o unidades retributivas son un tipo de incentivo económico que existen en el ámbito estatal desde 1995 y se utilizan como complemento remunerativo del Gabinete o bien como suplemento extraordinario para el personal estatal en general. A partir de la Decisión Administrativa 267/2018, se estableció que las unidades retributivas se debían dirigir al personal de gabinete y al personal de la Administración Pública Nacional bajo ciertas condiciones: primero, era necesario que no estuvieran contratados bajo modalidad de prestación de servicios personales u obras y/o asistencia técnica y/o becas. Segundo, su asignación podía ser tanto de manera regular como extraordinaria con la finalidad de premiar la iniciativa, el mérito o la productividad en el desempeño.

Esta segmentación y criterios en la utilización de las UR impactó en la capacidad adquisitiva de los trabajadores, producto de la reorganización en sus especificaciones:

Las personas que tenían unidades retributivas se les cortó, recién ahora están viniendo pero recortadas, bueno, también UPCN tienen unas paritarias muy malas y también hubo un deterioro muy fuerte del poder adquisitivo de las personas (Funcionario de Producción)

Por las características del sistema compensatorio estatal, estas UR se mostraron como la única estrategia financiera que tenía mayores posibilidades de responder al criterio meritocrático:

Solamente hay algunos sistemas que tenemos cierta discrecionalidad, que son lo que se llaman unidades retributivas, que lo que uno trata de hacer 
es, en algunos casos, compensar y tratar de mejorar en aquellos que están en cada unidad, donde el Secretario puede manejarla y bueno tratar de compensar aquellos que vos crees que son buenos y los querés mantener para que no se te vayan al sector privado o aquellos que han hecho la mejor tarea, esos temas son limitados. (Funcionario de Producción)

Pero así como se trataba de una herramienta compensatoria promotora de ciertas competencias, paradójicamente no podían utilizarse de manera descentralizada y su instrumentación estuvo al alcance, únicamente, de quienes ostentaban un cargo de Secretario. Esto llevó a que se considere que el manejo de las UR no haya sido lo suficientemente efectivo como para poder establecer un sistema meritocrático que promoviera la eficiencia y eficacia en los trabajadores de esas reparticiones:

Las UR primero que es como para los que lo tienen es casi un interés adquirido, sacarlas es un tema. Siempre te quedas corto porque son poquitas, además había UR que gravaban sueldos, no eran como bonus, vos sacas la UR y te quedaba el tipo [pone cara de asombro], eran remunerativas, por lo cual no había nada, por lo menos en mi área. Eso es un tema porque es muy difícil poder premiar, me hubiera encantado que exista como un bonus en el sector privado, para que realmente sea por mérito. (Funcionario de Producción)

Esta limitación, sumada a la complejidad que representaba el poder modificar las bases de la estructura salarial y los parámetros de ascenso y promoción del personal del Estado (SINAPA), generó que los mismos Altos Funcionarios concibieran como insuficiente el alcance de las estrategias remunerativas:

Lo que pasa es que al no haber un programa de incentivos $y$ reconocimiento, trabajar o no trabajar, hacer bien o no las cosas no tiene recompensa, entonces yo creo que eso sí es muy importante de forma centralizada, una estrategia de planificación de carrera, de ascenso, de reconocimiento, flexibilizar un poco más lo público (Funcionario de Producción) 


\section{Reconocimientos no monetarios}

Al contar con reducidas herramientas de reconocimiento financiero que les permitieran articular un entorno meritocrático, los Altos Funcionarios desplegaron una serie de intervenciones no monetarias dentro de ambas reparticiones. Estas maniobras se implementaron como alternativa para premiar a aquellos trabajadores cuyo comportamiento se vinculara con los criterios de agilidad, eficiencia y eficacia que requería ser cumplida por un servidor público.

La confianza, la flexibilidad y la libertad fueron otorgadas como recompensa en caso de que el trabajador estatal cumpliera con sus labores de manera eficiente y, entonces, lo merezca. Combinando intervenciones tanto en el plano individual como intra-grupal, se evaluaban los rendimientos de cada trabajador bajo parámetros internos (no vinculados con el sistema de evaluación vigente estatal) y estos premios se otorgaban en base a los resultados obtenidos. La característica principal de este tipo de estrategias es que no estaban sistematizadas, sino que cada repartición las implementaba internamente sin ningún lineamiento o procedimiento que fuera dictaminado por el Ministerio de Modernización o el área de Recursos Humanos de la dependencia:

Dando libertad, tanto a los coordinadores como respetándoles su espacio, demostrándoles que confío en ellos y que les doy la libertad de criterio para opinar distinto, siempre remarcando que me corrijan o que si veían algo que no coincida, que lo digan. Les doy mucha libertad, que por ahí no es tanto que hay que darle, pero respetándola. Después otra forma que parece menor, reconociendo las cosas en sí también, felicitando, diciéndole también a [otro funcionario], que lo están haciendo muy bien y después también comunicarle al otro que lo comunicaste, como lo hicieron con el Ministro. Creo que eso no es plata, pero por lo menos digo, 'hice las cosas bien, laburo bien y eso también está bueno que me lo reconozcan por lo menos de otro modo'. Al momento de 'necesito un día para hacer una cosa', o me 'pasó tal otra', que veo que laburan bien y demás siempre fui muy flexible, no así con los otros. (Funcionario de Producción) 
Otro tipo de estrategia de reconocimiento no monetario se tradujo en la utilización de desayunos o encuentros con las principales autoridades del Poder Ejecutivo. Estas reuniones respondían al intento de replicar una política muy comúnmente utilizada en el ámbito managerial, conocida como Método de Puertas Abiertas, que implica el crear un puente donde la línea operativa pueda estar en contacto directo con los altos mandos de una organización. Esta metodología tiene como propósito el promover un intercambio comunicacional más fluido y menos rígido, donde tanto los empleados y los mandos jerárquicos sean llamados por su nombre de pila para generar un entorno más informal. En sintonía con lo anterior, es muy común dentro de ambas reparticiones y en el discurso de quienes forman parte de Cambiemos, el tratar a los demás por su nombre de pila y no por su apellido.

Reuniones con el Presidente [de la Nación], desayunos conmigo, con el Ministro. (...). Poníamos mucha energía de compensación espiritual porque el Estado a veces no puede, es muy inflexible, tiene que ver con la cultura del trabajo. (Funcionario de Producción)

Eje 3: optimización del espacio y los procesos de trabajo

El aporte más valorado por los entrevistados, el cual consideran fue el más efectivo para la gestión de las personas, fue el relacionado con la modificación de los procesos de trabajo y el ambiente laboral. Las implementaciones de las diferentes herramientas tendientes a la digitalización de los procedimientos se mostraron como necesarias, relevantes y transformadoras de los procesos, con lo cual se consideró que al haber modificado la forma de ejecutar las operaciones cotidiana, se generó una mejora en las condiciones de trabajo. Esto, en consonancia con la dirección por objetivos, permitió establecer, según los Altos Funcionarios, un entorno promotor de la agilidad y la eficiencia institucional:

El hecho es, insisto, la mejora en su ámbito laboral, la mejora de los sistemas que ya el hecho de que los sistemas hacen que sea más eficiente el trabajo y antes la recriminación de que venían los usuarios y puteaban mejoró mucho, y ha hecho que mucha gente se empiece a sentir mejor en su ámbito 
de laburo y que se sienta mejor en su trabajo, como un poco más contenido y un poco más reconocido (Funcionario de Producción)

Hay alguien que dice que para cambiar la manera de ser o de trabajar la gente tenés que cambiar los procesos, más allá de las directivas e intenciones que puedas darles. (Funcionario de Hacienda)

Estas mejoras también fueron acompañadas de una reestructuración en los organigramas, eliminándose dependencias intermedias y reduciendo los niveles para obtener una estructura más reducida. Los parámetros por los cuales se realizaron estas modificaciones se vinculan con los criterios utilizados dentro del ámbito privado:

Achicamos, hicimos cambios de estructuras, de aplanar un poco. Había muchos entre el Ministro y el último empleado del último nivel. Había una cantidad de niveles muy grandes niveles comparados con los que hay en cualquier organización grande privada. (Funcionario de Hacienda)

Resulta interesante, también, el posicionamiento de los funcionarios de Producción y el lugar que tiene el ambiente de trabajo en términos de espacio físico propiamente dicho. Los entrevistados de esa dependencia en particular, cuando se referían al ámbito de trabajo y las modificaciones realizadas, manifestaban un gran sentimiento de orgullo:

Vos te recorres el Ministerio y ahí donde está Dante [Sica, el Ministro de Producción], el edificio es el mejor edificio público de Latinoamérica: cambiamos los ascensores, arreglamos el frente, cómo entras, todos los muebles. El edificio, los muebles, la silla ergonométrica, las computadoras, Fibertel [servicio de internet], la fibra óptica del internet. En el Call Center tengo los box de cada uno con un headphone, tienen que trabajar máximo 2 horas, después van [y] descansan. (Funcionario de Producción)

Para los funcionarios de esa dependencia en particular, el contar con un espacio físico considerado moderno, con un estilo que replicaba el de las grandes corporaciones y con comodidades similares, era un factor que también influía en la productividad y el bienestar de los trabajadores. 


\section{Conclusiones}

Se ha podido evidenciar, a lo largo del presente trabajo, que durante el período 2015 a 2019 se implementaron una serie de dispositivos tendientes a promover una transformación del Estado Nacional hacia un Estado, en términos manageriales, moderno. Estas acciones, combinadas con diferentes estrategias que permitieran potenciar sus ventajas, tuvieron como objetivo el poder re-orientar la conducta de los trabajadores estatales, determinando y controlando las diferentes formas de ser, pensar y sentir el rol laboral, pasando de ser simples "trabajadores del Estado" a "Servidores públicos".

Esta implementación y el éxito en la consecución de su objetivo principal, según se pudo evidenciar, dependía de la aceptación e internalización de ciertos dispositivos por parte de uno de los actores más complejos de dirigir, no por sus características en sí sino por su mera condición humana y no artificial: los trabajadores de la Administración Pública Nacional. Los devenidos "servidores públicos" eran considerados en lo discursivo como los principales protagonistas del plan, con lo cual parte del proyecto modernizador se enfocó en la gestión de los recursos humanos del Estado. Brindar un proyecto de desarrollo y carrera, establecer acciones de formación y capacitación permanente, así como también de reconocimiento en el desempeño, como parte de los dispositivos explícitos implementados, fueron parte de las estrategias necesarias para dirigir y orientar a los empleados públicos hacia los valores del nuevo paradigma institucional.

Producto de los resultados obtenidos en esta etapa de investigación, se pudo evidenciar que la implementación de las acciones realizadas por estos directivos, si bien parecieron haber sido efectivas para agilizar procesos a nivel operativo, no pudieron ser acompañadas por una estrategia que contemplara la internalización integral de los cambios metodológicos por parte de los usuarios cotidianos de estos sistemas (los trabajadores estatales). Si bien parte de las acciones formativas buscaron acercar al empleado público en la cultura de lo ágil, la importancia del bienestar del ciudadano y el uso de las tecnologías, no existieron acciones de formación que buscaran adecuar actitudes o tendieran a moldear aspectos de la personalidad hacia conductas que se vinculen con la innovación, iniciativa y 
otras competencias relacionadas con el paradigma que implicaba concebir a un Estado como moderno e inteligente.

Considerando que el recurso humano fue valorado como el principal motor que impulsaba esta acción transformadora (al menos discursivamente), las estrategias resultaron ser insuficientes para lograr un proceso de desarrollo del trabajador estatal en sintonía con los parámetros manageriales y que permitieran afianzar un criterio meritocrático en los ascensos y recompensas del personal. Eso, consideramos, se debió a varios factores: la falta de reglamentación a nivel general, la inexistencia de normativa que permitiera centralizar las políticas de recursos humanos implementadas en cada Ministerio, la rigidez del sistema de promoción de carrera vigente del empleado público (SINAPA) y la inviabilidad de poder modificar su estructura en un corto plazo.

Para lograr conducir a los trabajadores estatales y poder impactar en su capital corporal, los Altos Funcionarios se vieron obligados a implementar estrategias alternativas, tanto monetarias como no remunerativas. Estas acciones, muchas de las cuales fueron replicadas por los entrevistados producto de sus experiencias previas en el ámbito privado, al no estar reglamentadas terminaron siendo temporales, con lo cual es altamente probable que se terminaran vinculando a un período o un funcionario en particular y no como acciones permanentes que traspasaran un período de gobierno determinado. La ausencia del peso de lo formal y el respaldo de normativas que afiancen las estrategias explícitas generó que estos dispositivos tuvieran un carácter pasajero, lo cual ha ido claramente en detrimento de la instalación de dispositivos del tipo implícitos, los cuales seguramente impactarían de manera más profunda tanto en el cuerpo social como subjetivo de los trabajadores y hubieran permitido desplegar el ethos managerial en su máximo esplendor. A lo largo de las entrevistas, efectivamente, no se pudo pesquisar la existencia de ninguno de los dispositivos informales utilizados en las organizaciones manageriales.

Por último, y considerando el punto de vista de los entrevistados, el desafío que se les planteó a estos actores superó las habilidades con las que contaban. Confiados en el peso de sus habilidades y experiencias previas y munidos de una personalidad narcisista que iba en búsqueda de una redención absoluta, con pasión y con devoción, los managers fueron 
seducidos a formar parte del "cambio" que el Estado requería para aggionarse a los nuevos requerimientos del mundo, en términos de tecnología, procesos y competencias.

El rumbo transformador que se le intentó dar al Estado requería poder instrumentar estrategias para pasar de ser una institución atravesada por la «burocracia estructural» hacia un Estado «moderno» que sea «simple, ágil, confiable, cercano, transparente, participativo y, sobre todo, inteligente» (Clusellas et al, 2019). En el caso de los sujetos designados para instrumentarlas, la ausencia de experiencia en el ámbito público, el escaso o nulo conocimiento de los procesos internos, los prejuicios negativos previos respecto del trabajador estatal y de la dinámica de lo público, entre otros factores, incidieron en el propio proceso de aprendizaje de esos funcionarios. Al encontrarse con la realidad de ser parte del Estado, con una estructura y dinámicas que superaban las más complejas que hasta el más experimentado de los managers haya manejado, estos individuos debieron invertir un tiempo considerable para poder reconducir su propio capital corporal, rever sus propios modos de sentir y aprehender, aunque sea de manera incipiente, la magnitud y complejidad de una institución como lo es un Estado Nacional. Este tiempo invertido puede ser visto como un aspecto positivo en lo que respecta a la internalización de la realidad organizacional, su dinámica cotidiana y los valores que caracterizan a su cultura. No obstante, desde un punto de vista político, puede concebirse como contraproducente: la lógica que atraviesa a un puesto de alto mando estatal está signada por la incertidumbre, algo que si bien los managers conocen y manejan muy bien en el mundo de la empresa privada, en el mundo estatal la dinámica, ha quedado demostrado, es muy diferente. La carrera estatal y los factores que determinan el ascenso y la promoción en el Estado del personal no jerárquico son completamente antagónicos a los determinados en las grandes corporaciones, con lo cual los managers partieron de la base de una situación muy distante a la que estaban cómodamente acostumbrados a dirigir. Como bien mencionó uno de los entrevistados: «es difícil manejar una organización en la que los permanentes son los empleados y los que cambian son los de arriba».

La subjetividad estatal, estereotipada negativamente por el paradigma managerial, fue abordada utilizando estrategias comúnmente empleadas, conocidas y de probado éxito en las grandes empresas. Bajo una mirada simplista, se subestimó la complejidad que atraviesa y determina los distintos modos de sentir, pensar y vivir el Estado, lo cual llevó a que se 
implementen una serie de dispositivos que terminaron siendo insuficientes para lograr modernizar al Estado a través del cambio de la mentalidad y sentimientos de los trabajadores.

El desear implementar una ideología, una metodología, una práctica o un procedimiento de trabajo que comúnmente se utiliza en el ámbito privado y aplicarlo sin comprender los distintos aspectos que hacen al funcionamiento de una institución pública, pudo haber sido entonces un factor que incidió negativamente y fue en detrimento del éxito del plan modernizador del gobierno de la coalición Cambiemos.

\section{Referencias bibliográficas}

Aubert, N. y de Gaulejac, V. (1993) El coste de la excelencia. Barcelona, Paidós.

Bellomo, S. (2017). Entrevista. Apuntes para la modernización del Estado. Año 1, (1): 4.

Bernazza, C. (2016). Bienvenidos al pasado. Revista Perspectivas de Políticas Públicas. 6 (11): 23-37.

Blutman, G. y Hoya, A. (2019). Reinventando la rueda-cuadrada: el empleo público en la modernización del Estado Argentino (2015-2019). Red Revista Eletrônica de Administração. 25 (2): 40-62.

Boltanski, L. y Chiapello, E. (2002). El nuevo espíritu del capitalismo. Madrid: Akal.

Bordieu, P. (2000). Cosas dichas. Barcelona: Gedisa

Castellani, A (2017). Primer informe de investigación. Observatorio de las elites. UNSAM: Buenos Aires.

Clusellas, P., Martelli, E. y Martelo, M. (2019). Un gobierno inteligente: el cambio de la Administración Pública de la Nación Argentina 2016-2019. CABA: Boletín Oficial. 
Farías, M.L. (2021). La managerialización de las relaciones estatales en Argentina (20152019): ¿Cambiemos logró cambiarnos?. Innovar 31(79), 47-59. https://doi. org/10.15446/innovar.v31n79.91895

Farías, M.L. (2020). Un "Estado inteligente" al servicio del ciudadano: la transformación del Estado Nacional y la modernización de los trabajadores del Estado durante el gobierno de "Cambiemos". Ponencia presentada en III Jornadas de Estudios Organizacionales. UBA. 24 al 27 de noviembre de 2020, CABA.

García Fanlo, L. (2011). ¿Qué es un dispositivo?: Foucault, Deleuze, Agamben. A parte Rei 74. Extraído de http://serbal.pntic.mec.es/AParteRei/page84.html

Guerra Manzo, E. (2010). Las teorías sociológicas de Pierre Bourdieu y Norbert Elias: los conceptos de campo social y habitus. Estudios Sociológicos XXVIII (83): 383-409. El Colegio de México, A.C: México.

Hernández Sampieri, R., Fernández Collado, C. y Lucio, P. (2006). Metodología de la investigación. Buenos Aires: Mc Graw Hill.

Ibarra, A. (2017). Estamos construyendo un Estado transparente, innovador, ágil y cercano. Revista Aportes para el Estado y la Administración Gubernamental 33: 7-14.

Luci, F. (2012a). El management como gramática: la producción de dirigentes de empresas. Rev. Ciencias Sociales 135-136: 171-183.

Luci, F (2012b). La educación de los dirigentes de empresas: la formación en negocios y el acceso a la cúpula de las principales organizaciones. En Gessaghi, V. y Ziegler, S. (Comp.). La Formación de las elites. Investigaciones y debates en Argentina, Brasil y Francia (227-247). Buenos Aires: Manantial-Flacso. 
Pierbattisti, D. (2013). France Télécom y la internacionalización del management: la privatización de las telecomunicaciones en Francia y la Argentina (1990-2011). Revista Trabajo y Sociedad 21: 67-89.

Reynares, J. (2017). Neoliberalismo y actores políticos en la Argentina contemporánea. Perfiles latinoamericanos 25 (50): 279-299.

Scribano, A. (2012). Sociología de los cuerpos/emociones. Revista Latinoamericana de Estudios sobre Cuerpos, Emociones y Sociedad - RELACES 10 (4): 93-113.

Vasilachis de Gialdino, I. (2007). Estrategias de investigación cualitativa. Buenos Aires: Gedisa Editorial.

Vommaro, G. (2017). La larga marcha de Cambiemos. Buenos Aires: Siglo XXI.

Zangaro, M. (2011). Subjetividad y trabajo: una lectura foucaultiana del management. Buenos Aires: Herramienta.

\section{Notas}

1 Sigla de Ente Nacional de Telecomunicaciones.

2 Tomaremos como base el concepto de "espacio social" de Bordieu, concibiendo al mismo como un espacio donde las prácticas de los sujetos tienden a ajustarse espontáneamente a las posiciones sociales establecidas entre las mismas (Guerra Manzo, E., 2010: 397).

3 El autor comenta que se buscó analogar el accionar del Gobierno Nacional anterior con las acciones implementadas por el gobierno venezolano y su impacto en la estructura socioeconómica y política de ese país.

4 Para García Fanlo (2011:7), un dispositivo es un régimen social productor de subjetividad que inscribe en los cuerpos reglas y procedimientos, o como bien lo menciona, es productor de sujetos-sujetados a un orden del discurso cuya estructura sostiene un régimen de verdad. 
5 Para Aubert y de Gaulejac (1993), la empresa utiliza la pasión para convertir a la organización en un objeto de adhesión y de esa manera, generar en el sujeto niveles de energía suficientes para potenciar el funcionamiento interno. Pero al mismo tiempo, surge una situación paradigmática dado que, como consecuencia de lo anterior, pueden producirse efectos devastadores producto del exceso de energía.

6 Un ejemplo de esto es la implementación del sistema GDE (Gestión Documental Electrónica). 7 Acorde a lo especificado por el Decreto 801/18, los Ministerios de Trabajo y Agroindustria fueron absorbidos por el Ministerio de Producción.

8 Tal como lo demuestra Luci (2012b) en su artículo, la formación especializada de los managers en ciertas entidades educativas es necesaria para acceder a posiciones jerárquicas dentro de la estructura empresarial.

9 Flora, la empleada pública" forma parte del sketch del actor argentino Antonio Gasalla, quien interpreta sarcásticamente a una recepcionsista de una institución pública 